\title{
A phase-fitted and amplification-fitted modified Runge-Kutta method of fourth order for periodic initial value problems
}

\begin{abstract}
A new Runge-Kutta method, with phase-fitted and amplification-fitted is constructed for solving first-order ordinary differential equations with periodic solutions. This new method is based on the Runge-Kutta 3/8 Rule with fourth algebraic order. In the numerical results the new method is compared with the existing method; which show that the new method is more efficient.
\end{abstract}

Keyword: Amplification-fitted; Periodic solutions; Phase-fitted; Runge-Kutta methods 